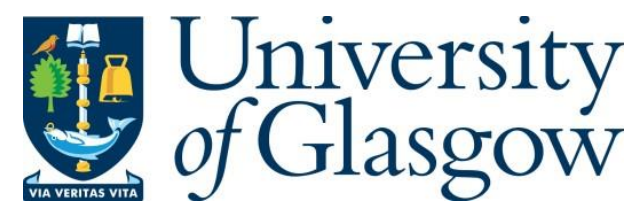

Raza, M., Le-minh, H., Aslam, N. and Hussain, S. (2018) A novel MAC proposal for critical and emergency communications in industrial wireless sensor networks.

Computers and Electrical Engineering, 72, pp. 976-989.

There may be differences between this version and the published version. You are advised to consult the publisher's version if you wish to cite from it.

http://eprints.gla.ac.uk/158481/

Deposited on: 6 March 2018

Enlighten - Research publications by members of the University of Glasgow http://eprints.gla.ac.uk 


\title{
A Novel MAC proposal for Critical and Emergency Communications in Industrial Wireless Sensor Networks
}

\author{
Mohsin Raza ${ }^{\mathrm{a}, \mathrm{b}}$, Hoa Le-minh ${ }^{\mathrm{b}}$, Nauman Aslam ${ }^{\mathrm{b}}$, Sajjad Hussain $^{\mathrm{c}}$ \\ ${ }^{a}$ Middlesex University, London \\ ${ }^{b}$ Faculty of Engineering and Environment, Northumbria Univeristy, Newcastle \\ ${ }^{c}$ School of Engineering, University of Glasgow
}

\begin{abstract}
In this paper, three MAC protocols, EE-MAC, CF-MAC and OD-MAC are proposed. EE-MAC incorporates emergency communication and allows immediate channel access for emergency traffic. CF-MAC compensates regulatory and open-loop control traffic and introduces a prioritized access for high priority nodes. It also allows nodes with critical data to reserve communication slots using control channel. Whereas OD-MAC presents a deadline based dynamic scheduler which ensures timely delivery of time-critical information. The paper presents mathematical modelling of the proposed protocols. For evaluation purposes, the performance of the proposed protocols is compared to IEEE 802.15.4e LLDN. The results show that the proposed protocols offer up to $92 \%$ reduction in delay in emergency communications at the cost of $5 \%$ to $15 \%$ increase in delay of time-insensitive data. A $60 \%$ and $85 \%$ reduction was observed in the channel access delay for regulatory and open-loop control traffic respectively along with notable improvements in communications reliability.

Keywords: MAC, real-time, wireless sensor network (WSN), reliability, hybrid, automation, industrial wireless sensor network (IWSN), TDMA
\end{abstract}

\footnotetext{
Email addresses: mohsinraza119@gmail.com (Mohsin Raza), hoa.le-minh@northumbria.ac.uk (Hoa Le-minh), nauman.aslam@northumbria.ac.uk (Nauman Aslam), sajjad.hussain@glasgow.ac.uk (Sajjad Hussain)
} 


\section{Introduction}

Wireless Sensor Networks (WSNs) formulate a communication network of spatially distributed autonomous sensor nodes. The wireless nodes used to form the network are equipped with microprocessor, memory, sensor board, radio, battery, secondary power sources and I/O interfaces. This allows wireless nodes in the network to form intelligent communication infrastructure. The cost efficiency, flexibility, self-healing abilities, localized processing and interoperability encourages use of WSNs in wide variety of monitoring, automation, data accumulation, sensory feedback and process control applications.

10 On the contrary to traditional WSNs, Industrial Wireless Sensor Networks (IWSNs) focus on critical and time sensitive industrial applications. In recent years, IWSNs have emerged as effective and cost-efficient solution for industrial automation and process control [1, 2, IWSNs, in comparison to some wired networks (with cabling costs of up to $€ 4337$ per meter [3]), offer a very nominal and cost-efficient, reconfigurable solution. However, the critical nature of industries, urge for higher reliability and real-time data delivery [4, 5]. Many industrial communication protocols are introduced to overcome reliability and latency issues Some of the prominent industrial protocols include Zigbee, WirelessHART, ISA100.11a, Wia-PA and 6LowPAN 9, 10, 11. Apart from these, two IEEE standards, IEEE 802.15.4 [12] and IEEE 802.15.4e [13, were introduced, where these standards served as a baseline for defining the PHY and MAC layer for various industrial protocols.

The IEEE802.15.4 uses Carrier Sense Multiple Access/Collision Avoidance (CSMA/CA). Although CSMA/CA based access schemes offer great potential

25 for low delays but guaranteed channel access is usually compromised due to collisions and lack of reliable channel access scheme. To address the reliability issues, IEEE 802.15.4e uses Time Division Multiple Access (TDMA). IEEE 802.15.4e primarily targets industrial applications to offer reliable and timely channel access for critical nodes. However, the pre specified access in TDMA, also introduces unwanted delay in time sensitive and asynchronous applications. 
Table 1: Communication Requirements in Industrial Systems

\begin{tabular}{lll} 
Traffic Category & Reliability & Time constraint \\
Safety / Emergency Traffic [6, 7, 8, & High & Few milliseconds \\
Regulatory control traffic [6, 8, & High & Tens of milliseconds \\
Supervisory control Traffic [6, 8, & High & Tens of milliseconds \\
Open loop control traffic [6] & Medium & Seconds-minutes \\
Alerting traffic [6, 8, & Medium to low & Seconds-minutes \\
Monitoring traffic [6, 7, 8, & Low & minutes to hours \\
\hline
\end{tabular}

Due to critical nature of industrial applications, IWSNs must ensure certain time and reliability bounds $[5]$. According to the International Society of Automation (ISA), the industrial systems can be distributed into six classes [6] based on the access schemes, reliability and latency requirements. The communication 35 requirements for these systems are listed in Table 1. To address the issues of different classes in industrial automation, three MAC protocols are proposed.

i EE-MAC introduces optimized channel access for emergency communications and provides immediate channel access to critical nodes. Initial results of this protocol are published in IEEE conference, CCODE 2017 [14].

40 ii CF-MAC optimizes communication in regulatory, open-loop and supervisory control systems. It provides communication failure compensation for regulatory control systems as well as schedules asynchronous channel access requests for supervisory control systems.

iii OD-MAC incorporates deadline based scheduling of nodes in the network.

45 The rest of the paper is organized as follows. Section 2 presents literature review. System model is presented in Section 3. Section 4 discusses the results and presents performance analysis. Finally, Section 5 gives conclusion and future directives. 


\section{Literature Review}

50 work 13 which limits the maximum delay in consecutive channel accesses of a node to 10 milliseconds 9]. Although IEEE802.15.4e LLDN and some other industrial protocols (WirelessHART and ISA100.11a), by incorporating TDMA, offer better reliability, yet, the slotted access, adds unwanted delay. CSMA/CA based channel access which adds uncertainty. In [15], a priority based MAC scheme is presented which considers four priority levels. In this scheme high priority traffic is allowed to overtake low priority node's communication slots which induces extended delays in low priority traffic. Furthermore, attempt to access the low priority nodes timeslot. In [16], authors define an arbitration based MAC, where the preassigned arbitration frequency is used to provide the prioritized access to the channel. In this protocol, each node has to wait for arbitration request period to request channel. The protocol also requires special coordinator to process orthogonal frequencies.

\section{System Model}

In the proposed system, TDMA based channel access scheme is used with hierarchical architecture to ensure a maximum two hop delay from sensor node to the control centre. The communication between the sensor nodes and the relevant coordinator node uses a star topology. The communication between the coordinator nodes and the control centre uses multi-channel access scheme to establish parallel data streams. Nodes'association takes place prior to communication scheduling and is facilitated by control channel. During setup phase, potential cluster-heads are nominated. The nominations depend on energy avail75 ability and suitability of location of the nodes. If the traffic requirements increase, the inactive cluster-heads may also be activated, forming smaller, dense 

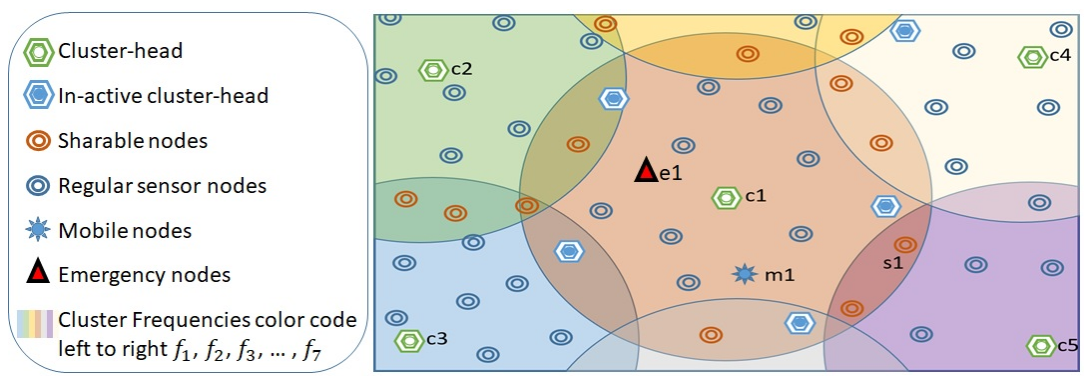

Figure 1: Graphical representation of cluster based IWSNs

coverage networks (see Figure 11. Since the industrial applications are primary focus of this work, which generally have static networks, therefore, mobile nodes are mostly no-existent in such environments. However, to facilitate mobility, the control channel based affiliation is facilitated where new nodes can associate with a particular cluster-head. If the already associated nodes in the cluster reaches to maximum allowable limit, a resource sharing mechanism is implemented where nodes common to two or more clusters can be transferred to cluster with lesser load.

85 An example scenario is presented in Figure 1 where multiple clusters are shown each using frequency $f_{i}$. Cluster 1 , with cluster-head $\mathrm{c} 1$ is facilitating maximum number of nodes. As the channel request is made from the mobile node m1, the sharable nodes will be exchanged between cluster 1 and cluster 5 to allow c1 to facilitate mobile node. If the load significantly increases the inactive cluster heads can also be activated.

\section{1. $E E-M A C$}

Emergency Enabled MAC (EE-MAC) allows nodes with critical/emergency information to request channel access. Since the occurrence of emergency communications is asynchronous in nature so a hybrid scheme is introduced.

In case of emergency, a control channel based slotted request mechanism is introduced. It allows the coordinator to halt the regular transmission and initiate emergency communication by inserting time slots in TDMA frame. In case of multiple emergency requests, a queuing function is used to allocate the 
Table 2: Communication Requirements in Industrial Systems

\section{Parameters}

Time slots in LLDN superframe

Emergency nodes in a cluster

Packet Payload bits

Payload transmission time

Superframe time duration (LLDN)

Data Rate

Prob. of successful communication

Emergency traffic Arrival rate

Number of emergency requests

Time slot duration

Access Delay

Avg. successful communication delay

Communication window duration

Acknowledgement window duration

EE-MAC avg. added time-slots duration

Maximum additional slots in CF-MAC

Nodes communicating in Segment-i

Communication failure probability

No. of failed comms. per segment

Avg. comm. failures per segment

Prob. of Comm. failures in $s_{i}$

Sensor value

Setpoint, Threshold high/low

supervisory control time-slots

supervisory feedback sensor nodes

\%age channel request queries

Total nodes

Time deadline of node $\mathrm{i}$

OD-MAC added slots duration

Failed comms. in a superframe

Failed comms. in last superframe

\section{Variable(s)}

$n$

$m$

Payload_bits

PL_delay

$T_{L L D N}$

$R_{b}$

$p$

$\lambda$

$\alpha$

$t$

$d$

$d_{\text {succes }}$

$(1-\delta) \times t$

$\delta \times t$

$e$

$k$

$s_{i}$

$q /(1-p)$

$x$

$A_{f}$

$P\left(f_{s i}\right)$

$s$

$S p, T h_{\text {High }}, T h_{\text {Low }}$

$h$

$u$

$R_{s}$

z

$d l(i)$

$\Delta$

6
Value(s)

20

$1,2,10$

960 bits

$3.84 \mathrm{~ms}$

$10 \mathrm{~ms}$

$250 \mathrm{kbps}$

0.7-0.999

1-500

$\approx 300 \mu s$

$-$

$-$

$-$

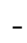

$-$

5

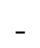

$-$
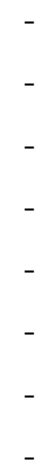

$-$

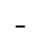

$-$ 


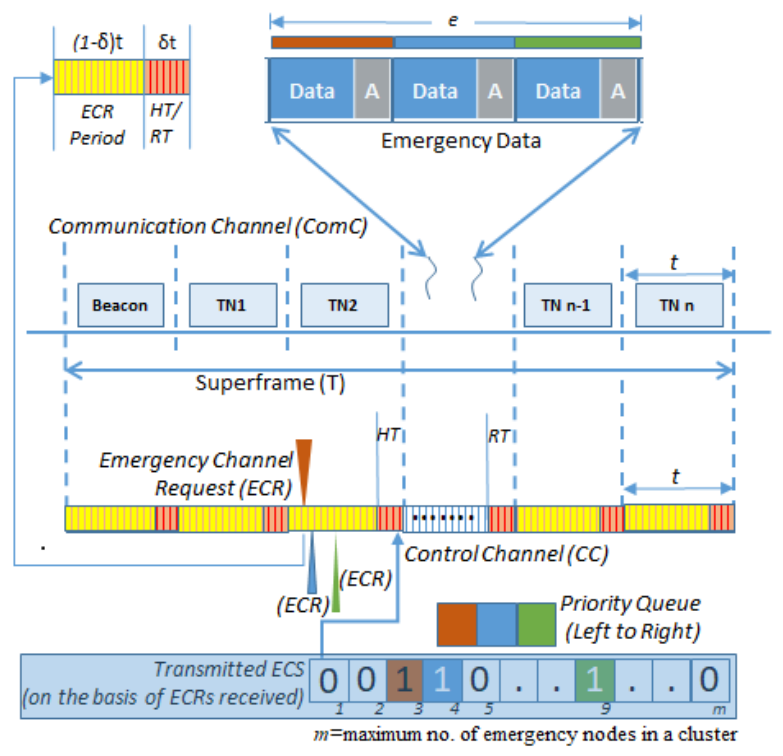

Figure 2: EE-MAC Operation

resources sequentially. For such cases the communication of regular TDMA can be stopped for multiple timeslots. To ensure the collision free transition, coordinator initiates halt $(H T)$ and reinitiate $(R T)$ sequences which informs the regular nodes to stop and resume communication when needed. A minimum halt duration is also included in $H T$ sequence to improve accuracy.

In Figure 2, superframe structure of EE-MAC and related details are presented. As represented in figure, each superframe is divided in $n$ timeslots, each of duration t. Each timeslot is further divided in communication and acknowledgement window of duration $(1-\delta) \times t$ and $\delta \times t$ respectively. In control channel, each time slot is divided in the Emergency Channel Request (ECR) period and halt/Reinitiate $(H T / R T)$ Period. A slotted access is used in ECR period where it is divided in $m$ slots. Each emergency node is allocated one of these $m$ slots to initiate ECR (Overlapping slot allocation among the emergency nodes can also be used to accommodate larger number of emergency nodes). After the completion of the ECR period if one or more channel requests are received, the coordinator initiates $H T$ during $H T / R T$ period to halt the ongoing regu- 
lar communication. By default, the regular communication is stopped for single time slot but if more than one emergency communication requests were received during ECR period, the halt duration is extended accordingly and this duration is communicated during $H T$ sequence transmission along with the Emergency Communication Sequence (ECS) (See Figure 2 for the ECS based on the ECRs received in the ECR period). This allows the regular communication nodes to go to sleep mode for the halt duration (HD) to conserve energy. Table II lists variables/parameters along with their selected values. The performance of EE-MAC is compared with IEEE 802.15.4e LLDN. Mathematical model for the proposed EE-MAC protocol is also presented.

The superframe duration of IEEE $802.15 .4 \mathrm{e} L \mathrm{LDN}$ is given by $T_{L L D N}$, whereas superframe duration of EE-MAC is given by

$$
T_{E E-M A C}=T_{L L D N}+e
$$

Where $e$ is the average duration ( \pm deviation $(\Delta)$ from mean) of additional timeslots added to the superframe to compensate emergency communications. Since the emergency communication in industrial environments is asynchronous and event driven, therefore it is modelled as a Poisson $(\alpha)$ distribution. The duration of timeslot $(t)$ is presented in Eq. 2 whereas the PMF of the Emergency occurrences $(x)$ in ECR period $(1-\delta t)$ is presented in Eq. 3 .

$$
\begin{aligned}
t & =\frac{T_{L L D N}-M A C \_ \text {Payload }}{n} \\
P_{X}(x) & = \begin{cases}\frac{\frac{\alpha^{x} e^{\alpha}}{x !}}{\sum_{y=0}^{m} \frac{\alpha^{y} e^{\alpha}}{y !}}, & \text { if } x=0,1,2, \ldots m \\
0, & \text { otherwise }\end{cases}
\end{aligned}
$$

Here $m$ is the number of emergency nodes in a cluster and $\alpha=\lambda \times T_{L L D N}$. The average time duration of added emergency slots within a superframe is given by

$$
e=t \times \sum_{x=0}^{m}\left(x \times \frac{\frac{\alpha^{x} e^{\alpha}}{x !}}{\sum_{y=0}^{m} \frac{\alpha^{y} e^{\alpha}}{y !}}\right) \pm\left(\sum_{x=0}^{m}\left(x-\left(x \times \frac{\frac{\alpha^{x} e^{\alpha}}{x !}}{\sum_{y=0}^{m} \frac{\alpha^{y} e^{\alpha}}{y !}}\right)\right)^{2} \times P_{X}(x)\right)
$$


The occurrence time of the emergency communications is modelled as uniform distribution. For evaluation purposes, the access delay, time between emergency channel request to transmission, is presented in Eq. 5 and Eq. 6, for both IEEE802.15.4e LLDN and EE-MAC respectively.

$$
\begin{gathered}
d_{L L D N}=\frac{1}{2} T_{L L D N} \\
d_{E E-M A C}=\sum x=1^{m}\left[\left(\delta t+\frac{1}{2} t+(x-1) \times t+\left(\frac{x}{n} \times P L \_ \text {delay }\right)\right) \times P_{X}(x)\right]
\end{gathered}
$$

In emergency communications, average delay between emergency request and successful transmission for IEEE 802.15.4e LLDN (d_success $\left.s_{L L D N}\right)$ and EEMAC (d_success $\left.s_{E-M A C}\right)$ is presented in Eq. 7 and Eq. 8 respectively.

$$
\begin{gathered}
d_{\_} \text {success }_{L L D N}=d_{L L D N} \times \sum_{w=1}^{\infty} w \times p(1-p)^{w-1} \\
d_{\_s u c c e s s_{E E-M A C}}=d_{E E-M A C} \times \sum_{w=1}^{\infty} w \times p(1-p)^{w-1}
\end{gathered}
$$

Here $p$ is the probability of successful transmission and $w$ is the count of transmissions until the successful communication takes place.

The performance of this protocol is evaluated for emergency systems in terms of channel access delay and average successful communication delay; details of which are presented in Section 4.

\section{2. $C F-M A C$}

Critical feedback MAC (CF-MAC) takes in consideration the critical feedback information in close-loop regulatory control systems, open-loop control systems and close-loop supervisory systems. Due to the relatively different requirements of the above-mentioned application areas, the operation of this protocol is divided in two cases depending on the targeted objectives. 


\subsubsection{Failure compensation}

In feedback control systems, the protocol allows the retransmission of the failed communication to improve the system reliability as well as time bounded delivery of critical information. Since regulatory control systems require synchronous feedback for controlled operation of the process, therefore, the number of retransmission slots that can be inserted in a superframe are limited to restrict the maximum delay between two consecutive transmissions of sensory nodes. To ensure minimal delay, the time duration of a superframe is limited to $10 \mathrm{~ms}$, which can only be extended by a duration no more than $\sigma T_{L L D N}$, (where $\sigma$ defines the allowable percentage increase in the delay of two consecutive transmissions of a critical node). For simplicity, the extension in the duration of superframe will be referred as $k$, the number of additional time-slots that can be added in the superframe, where $k=f\left(\sigma, T_{L L D N}\right)$. The addition of time-slots in the superframe is initiated by the cluster-head on unsuccessful communication from sensor nodes. The time-slots are added at the end of a transmission sequence/segment as represented in Figure 3. $H T$ and $R T$ sequences are used to halt and re-initiate regular transmission. The communication in a superframe is divided in four priority levels, to facilitate communication of regulatory control, open-loop control, supervisory control and monitoring systems. The superframe with priority wise segmentation is represented in Figure 3 . If the transmission of one or more nodes in a particular segment fails, the additional slots for retransmission are added after the segment ends. The maximum allowable additional slots are filled with the retransmission of the highest priority nodes first. The mathematical modelling of the key performance attributes of the proposed protocol is presented as follows.

The probability of failure in communication of nodes in a particular segment is modelled as a binomial distribution and is presented in Eq. 9 whereas the average communication failures per segment is presented in Eq. 10.

$$
P(x)=\left(\begin{array}{c}
s_{i} \\
x
\end{array}\right) q^{x}(1-q)^{s_{i}-x}
$$

Here $s_{i}$ is the number of nodes communicating in segment- $i, x$ is the total 


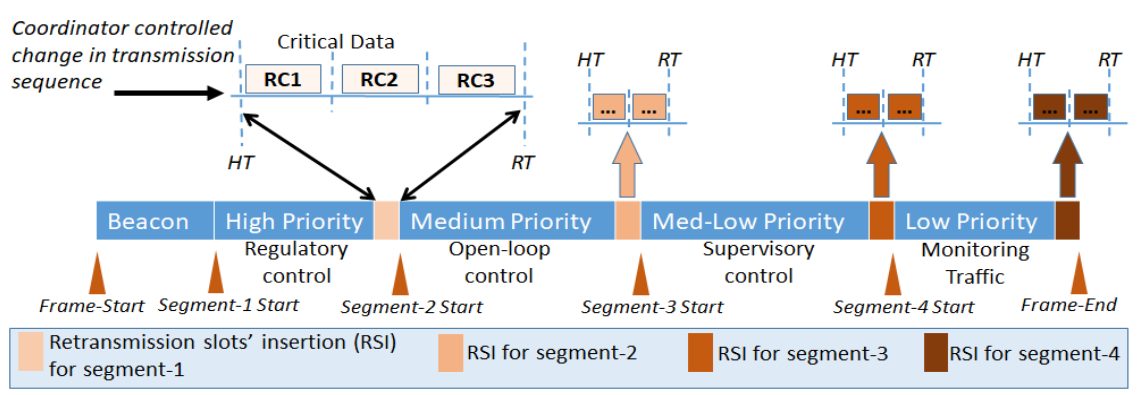

Figure 3: CF-MAC Superframe

number of failed communications and $q$ is the communication failure probability of a single node.

$$
A_{f}(i)=\sum_{x=0}^{s_{i}} x \times\left(\begin{array}{c}
s_{i} \\
x
\end{array}\right) q^{x}(1-q)^{s_{i}-x}
$$

The CF-MAC allocates additional slots for retransmission, of failed communications which improves the reliability of the system as a whole. The probability of failure for segment-1 (Figure 3 High priority section), in CF-MAC is presented in Eq. 12 in contrast to IEEE802.15.4e LLDN, presented in Eq. 11.

$$
\begin{gathered}
P\left(f_{s 1}\right)_{L L D N}=\sum_{x=1}^{s_{1}}\left(\left(\begin{array}{c}
s_{1} \\
x
\end{array}\right) q^{x}(1-q)^{s_{1}-x}\right) \\
P\left(f_{s 1}\right)_{C F-M A C}=P\left(f_{s 1}\right)_{L L D N} \times \sum_{y=1}^{x}\left(\left(\begin{array}{l}
x \\
y
\end{array}\right) q^{y}(1-q)^{x-y}\right)+ \\
{\left[\sum_{x=k+1}^{s_{1}}\left(\left(\begin{array}{c}
s_{1} \\
x
\end{array}\right) q^{x}(1-q)^{s_{1}-x}\right)\right]}
\end{gathered}
$$

Since communications in segment-2 in the superframe (Figure 3) are also important, therefore, a relationship for probability of failure in segment-2 communication is presented in Eq. 13.

$$
\begin{array}{r}
P\left(f_{s 2}\right)_{C F-M A C}=\sum_{x=1}^{k}\left[\left(\left(\begin{array}{c}
s_{2} \\
x
\end{array}\right) q^{x}(1-q)^{s_{2}-x}\right) \times\left(\left(\sum_{y=1}^{x}\left(\begin{array}{l}
x \\
y
\end{array}\right) q^{y}(1-q)^{x-y}\right)+\right.\right. \\
\left.\left.\left(\sum_{z=k-x+1}^{k}\left(\begin{array}{c}
s_{1} \\
z
\end{array}\right) q^{z}(1-q)^{s_{1}-z}\right)\right)\right]+\left[\sum_{x=k+1}^{s_{1}}\left(\begin{array}{c}
s_{1} \\
x
\end{array}\right) q^{x}(1-q)^{s_{1}-x}\right]
\end{array}
$$


Using Eq. 11-13, the probability of successful communication of segment- $i$ is defined as $P\left(S_{s i}\right)=1-P\left(f_{s i}\right)$.

The probability of failure in segment-3 and segment-4 $\left(P\left(f_{s 3}\right)_{C F-M A C}\right.$ and $\left.P\left(f_{s 4}\right)_{C F-M A C}\right)$ can also be derived, however, segment-3, dedicated for supervisory control traffic is discussed in detail in Section 3.2.2 and due to the lesser significance of communication in segment-4, and marginal improvements introduced by CF-MAC in this segment, the effect is not thoroughly investigated. To further investigate the impact of insertion of additional time-slots in the superframe, the average channel access delay of segment-1 nodes after communication failure is modelled for both IEEE802.15.4e LLDN and CF-MAC and is presented in Eq. 14 and 15 respectively.

$$
\begin{gathered}
d_{L L D N_{-} s 1}=T_{L L D N} \\
d_{C F_{-} M A C}=\left[\left(s_{1} \times t\right) \times\left(\sum_{y=1}^{k}\left(\begin{array}{c}
s_{1} \\
y
\end{array}\right) q^{y}(1-q)^{s_{1}-y}\right)\right]+ \\
\\
{\left[T_{L L D N} \times \sum_{x=k+1}^{s_{1}}\left(\begin{array}{c}
s_{1} \\
x
\end{array}\right) q^{x}(1-q)^{s_{1}-x}\right]}
\end{gathered}
$$

\subsubsection{Asynchronous communication (CF-MAC)}

To facilitate asynchronous feedback, in supervisory control systems, nodes with critical control information can request a transmission slot using the control channel. The request can be initiated if a notable change in the sensor reading occurs and need to be reported. As an example, Figure 4 represents the sensor readings, where feedback control is established to maintain the readings between the specified range of sensor value, marked by green strip. In the figure, a steep drop can be seen for value of x-axis (Time) near 1000. Under such circumstances, sensor node requests coordinator for channel access, which is being dealt in a similar manner as in case of EE-MAC using control channel requests. However, while handling supervisory control information in CF-MAC, two main differences from EE-MAC can be observed. First, the initiated request 


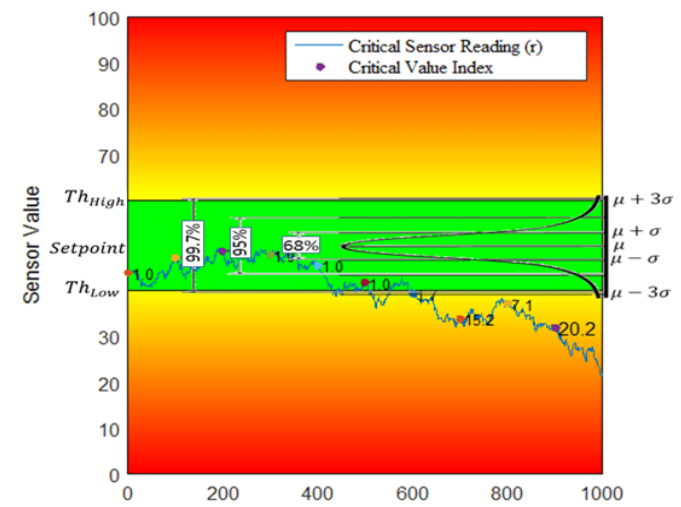

Figure 4: Sensor Readings

is not instantly addressed, rather, the coordinator/cluster-head will schedule the communication in segment-3 (see Figure 3). Second, maximum slots added in segment-3 are limited and unlike EE-MAC, due to lower priority of application at hand, communication requests from some nodes might be postponed to next superframe for communication. The coordinator in CF-MAC also implements queuing function, where requests are addressed sequentially based on the traffic priorities.

A careful modelling is needed for scheduling asynchronous transmission. Therefore, a probabilistic model is presented to specify the number of sensor nodes that can be served by a specific number of timeslots in the superframe. Further, to this, since no sensor node in segment-3 receive a dedicated time-slot, a thorough evaluation of slot to sensor ratio is established to provide desired Quality of Service (QoS). By ensuring careful modelling, frequent threshold violations can be avoided. The probability density function of system output for sensor reading is modelled as

$$
f(s)=\frac{1}{\sqrt{2 \pi \sigma^{2}}} e^{\frac{(x-\mu)^{2}}{2 \sigma^{2}}}
$$

Here $s$ is the sensor value where process control is modelled so that $S p=\mu$ and $S p \pm T h \approx \mu \pm 3 \sigma$. The specified condition ensures a relatively lower probability for control processes to violate the threshold. With the specified condition up-to $99.7 \%$ of the time the sensor readings will be within the specified thresholds i.e. 


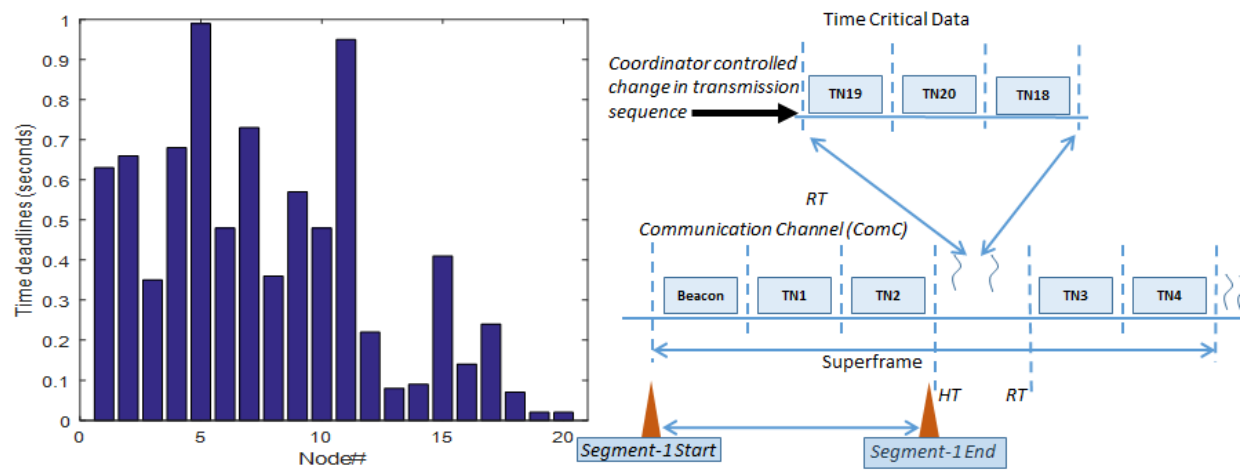

Figure 5: EE-MAC Operation

$$
P[S p-T h<s<S p+T h]>0.997
$$

Further to this, number of nodes accommodated in $h$ time-slots (dedicated for supervisory nodes) in superframe are represented by $u$ in Eq. 18 .

$$
\sum_{i=h+1}^{u}\left(\begin{array}{l}
u \\
i
\end{array}\right) r^{i}(1-r)^{u-i} \leq R_{s}
$$

here $r=1-P[S p-T h<s<S p+T h], u$ is number of supervisory feedback sensor nodes and $R_{s}$ is the desired percentage channel request queries per unit time exceeding the number of slots provided.

The performance of CF-MAC is evaluated in terms of reliability improvement and access delay for regulatory control. Whereas the network scalability under pre-specified QoS conditions is evaluated for supervisory control systems. A detailed discussion can be found in Section 4.

\section{3. $O D-M A C$}

OD-MAC targets two critical aspects of the industrial automation, deadline based scheduling and on-demand communication. The control channel is used for the information request from the nodes in the cluster. Since the communication is controlled by the coordinator, on demand communication can be initiated by the coordinator at any time. However, to keep the schedule as 
Input: $d l(1), d l(2), \ldots d l(z), f_{\text {stack }}, N, z$; Result: $N, f_{\text {stack }}$

while (1) do

timer0.start ()$; w=1 ; / /$ starting timer

for $i \leftarrow 1$ to $z$ do

| $s d(i)=\operatorname{sortAscending}(d l(i)) ; / /$ sorting nodes by deadlines

end

$S c h(1 \rightarrow N)=f_{\text {stack }} ; / /$ Scheduling failed communications

for $i \leftarrow N+1$ to $n+N$ do

$\operatorname{Sch}(i)=\operatorname{sd}(i-N)$;

end

$T_{O D-M A C}=T_{L L D N}+\Delta ; / /$ current duration of frame

Broadcast(sch/beacon); //broadcasting schedule

for $i \leftarrow 1$ to $n+N$ do

pkt.rcv $(c h, i)$; //receiving pkt from node $i$, channel $c h$

if $\mathrm{crc}=$ true then

ack; reset(deadline);

else

| $f_{\text {stack }}(w)=n_{\text {id }} ; w++; / /$ stacking failed comms.

end

if $i=$ ceil $\frac{n+N}{2} O R i=n+N \& w>1$ then

initiate $(H T) ;$ transmit $\left(f_{\text {stack }}\right) ;$ initiate $(R T) ; / *$ mid/end

of frame reached*/

end

end

for $i \leftarrow 1$ to $z$ do

| $d l(i)$-timer $0 / /$ remaining time till deadline reaches

end

timer0.reset ()$/ /$ resetting timer

end

Algorithm 1: Deadline based and on-demand communication scheduling 
predictable as possible, on demand communication is only initiated at the mid and end of the superframe, if needed. The communication in OD-MAC allows the coordinator to schedule the on-demand communications as well as deadline based communications. OD-MAC also introduces a deadline based scheduler, which manages the schedule of communication of the nodes and, where needed, alters the transmission sequence by inserting a special on demand transmission block. An example scenario is presented in Figure 5. It can be seen that a regular sequence of transmission is followed up to certain extent, however, due to the relatively short deadline of nodes 19, 20 and 18 (TN19, TN20 \& TN18, as represented in deadline bar graph in Figure 5], are specially accommodated. The deadline based scheduler allows application versatility and schedules load more effectively. The Algorithm for deadline based scheduling for heterogeneous sensing deadlines is presented in Algorithm 1 where the control channel based communication is used for communicating updated schedule at the start of the superframe.

The proposed scheduling algorithm formulates a schedule for every superframe communication based on the sensor deadlines and failures in the previous communications, as represented in Algorithm-1.

\subsection{Applicability of proposed protocols in industries}

In industrial environments various applications run simultaneously. These applications can belong to different classes of industrial systems and can have diverse requirements. An example scenario is presented to elaborate the working of the proposed protocols and how these protocols can assist in facilitating uninterrupted operation in the industries. As an example, a typical industry is considered which consists of three main classes of industrial systems: 1) critical and highly sensitive systems which are activated in case a major anomaly is occurred, 2) process control systems which introduce automation and process optimization and 3) monitoring systems to accumulate sensory data, to investigate plant efficiency and to analyse potential future improvement possibilities. The effective communication of traffic generated by these systems play an im- 
portant role in uninterrupted operation of the sensor nodes.

Emergency systems are usually triggered to minimize safety threats and lessen the severe consequences of uncontrolled changes in the automated systems to avoid operational and safety hazards. Emergency communications are one of the integral parts of emergency systems. These communications ensure timely delivery of critical information. Mostly such communications are isolated from the main communications and are provided dedicated channels which allow uninterrupted delivery of such messages to the control centre. Although the discussed solutions work, however, it requires dedicated communication bandwidth. Automation and process control systems require communication between sensory elements and the control centre. Traffic generated by such systems can either be synchronous, initiated after regular intervals (e.g. regulatory and open loop control systems) or asynchronous, generated whenever some thresholds are exceeded (e.g. supervisory control systems). Monitoring traffic is generated by time insensitive and less critical processes and information accumulated has no direct effect on the operations of industry, rather gives statistical values to evaluate bottlenecks in system and potentials for future improvements.

For the presented case scenario, consider Figure 1 where cluster-1 has nodes from all three systems present in the same vicinity. In such cases isolating emergency communication degrades systems performance. Apart from this, less careful communication scheduling may also cause unnecessary delays in regulatory and supervisory control communication. Communication failure can also have significant effect on reliability and real-time data delivery. To address these issues the proposed protocols can play an important role. EE-MAC allows embedding emergency traffic within the regular traffic and optimizes the bandwidth efficiency and coexistence of diverse processes. It also ensures that the channel is instantly allocated for emergency communication requests. CF-MAC optimizes priority based communication. It takes in to consideration priority of 225 different traffic types and reschedules communication accordingly. The scheme facilitates regulatory and open-loop control traffic communication within the specified time deadlines. It also ensures a suitable reliability in supervisory con- 


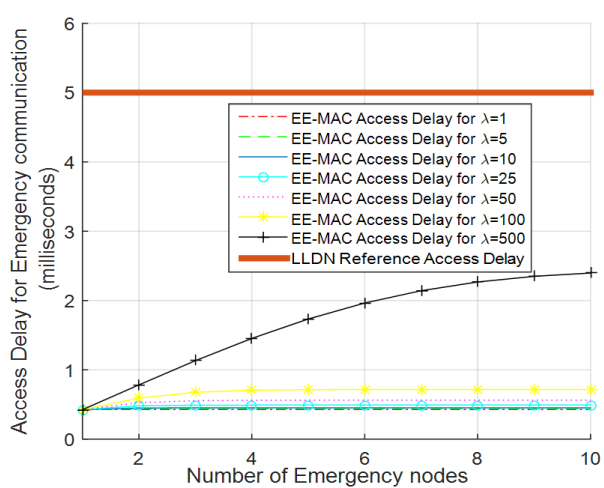

Figure 6: Channel access delay

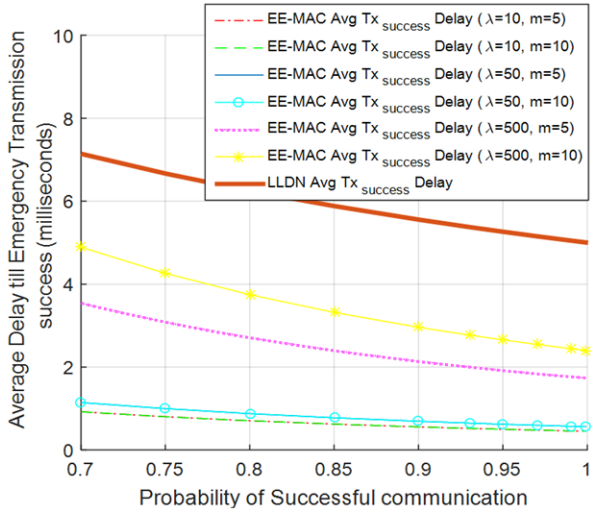

Figure 7: Average successful communication delay

trol traffic. In cases where traffic from multiple systems co-exist, the scheduling of such information is also a challenge. To schedule the communication from different communication systems while ensuring the time deadlines are met is the objective achieved by OD-MAC. All the three protocols collectively offer optimized channel access, priority based communication rescheduling and deadline based information scheduling.

\section{Results and Discussion}

The performance of the EE-MAC is judged in comparison to the IEEE802.15.4e LLDN, where the performance of EE-MAC is represented in terms of access delay, average timeframe extension and average delay till emergency communication to be successfully completed. In Figure 6, the access delay for IEEE802.15.4e LLDN and EE-MAC is presented as a function of number of emergency nodes. Since the LLDN offers a TDMA based access so the emergency nodes are provided with uniform access delay represented in the figure. On the other hand, EE-MAC provides on demand channel access, therefore the access delay for the EE-MAC is relatively lower to that of LLDN. Figure 6 shows that EE-MAC even under extreme conditions $(m=10, \lambda=500$ emergency communication requests per second (on average)) manages to offer a $50 \%$ reduction in the ac- 


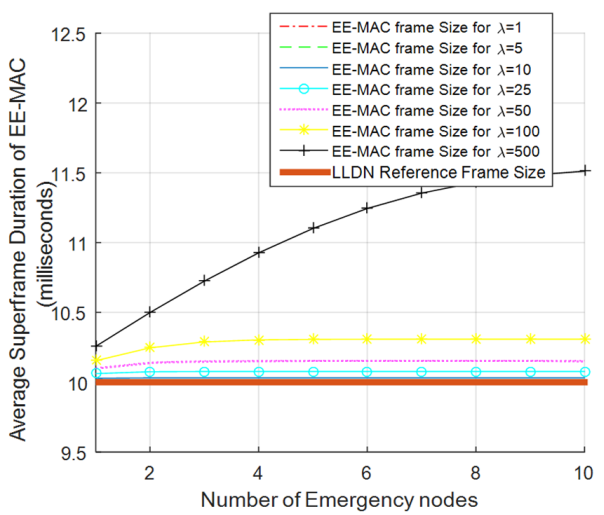

Figure 8: Average Duration of superframe

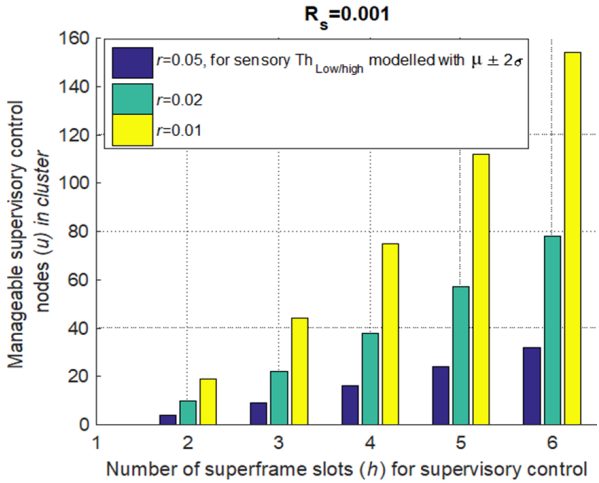

Figure 9: Supervisory control traffic timeslots $(h)$ to supervisory nodes $(u)$ ratio

cess delay. The average access delay for less extreme cases is evaluated to be under 0.6 milliseconds. The overall reduction in the access delay by EE-MAC can range from $50 \%$ to $60 \%$ for relatively extreme conditions and $88 \%$ to $92 \%$ for less extreme conditions.

To further investigate the delay in emergency communication, the average delay till successful communication for the different values of $\lambda$ (the number of emergency communication requests per second) and $m$ (number of emergency nodes) is presented in Figure 7. The figure shows that the EE-MAC under poor channel conditions $(p=0.7)$ and high number of emergency requests (500 requests per second, on average) still manages to reduce the average delay to 4.89 milliseconds. The average delay for less extreme cases is estimated to be less than 1.2 milliseconds. The presented analysis show that EE-MAC manages to reduce the average delay till the successful communication, of emergency information by $31 \%$ in extreme circumstances. However, in less extreme circumstances $(p>0.9 \& \lambda<100)$ delay reduces by $84 \%$ to $91.5 \%$. It can be seen that the access delay $(d)$ and average successful communication delay $\left(d_{\text {success }}\right)$ are not the same as the scheduling failures are also considered in the evaluation.

The improvements in the average delay of emergency communications was achieved with the insertion of additional slots to the MAC superframe, which 
extends the superframe duration and the average access delay of non-emergency communication. In Figure 8, the average superframe duration for both IEEE802.15.4e LLDN and EE-MAC is presented for different number of emergency nodes. It can be seen that the average access delay in EE-MAC is increased by 1.5 milliseconds for extreme conditions $(m=10, \lambda=500)$. Since the delay is added to the communication nodes with less stringent time constraints and an overall increase in average delay is below $7 \%$ for most of the cases which is considered non-critical. Apart from this a reduction of up to $92 \%$ in the emergency transmission delay is achieved.

275 The evaluation of the EE-MAC suggests that the use of control channel, to request a time-slot and extension of superframe duration effectively handles the emergency communication. It reduces the channel access delay and improves communication reliability for emergency traffic. However, due to the added delay $(\simeq 7 \%)$ in the regular communication, it is recommended that emergency traffic is isolated using different frequency channels where the monitoring traffic can be transmitted on the same channel. Overlapping emergency traffic with monitoring traffic allows up to $92 \%$ delay reduction in emergency traffic without having any notable impact on the monitoring traffic due to its immunity towards delay.

CF-MAC targets regulatory control traffic, open-loop control traffic, supervisory control, alerting, and monitoring traffic. The performance in regulatory and open-loop control is evaluated based on the reliability improvement and channel access delay in comparison to IEEE802.15.4e LLDN. Since LLDN uses no segmentation, a natural segmentation is established for comparison purposes. Furthermore, for evaluation purposes the maximum extension to the superframe duration in CF-MAC is defined by $k$, where $k$ is fixed to 5 .

Since, regulatory control, open-loop and monitoring traffic are periodic in nature so dedicated slots are scheduled in the superframe for each of these traffic types as represented in figure 3. Supervisory control and alerting traffic are asynchronous in nature, therefore, a careful modelling for these type of nodes 


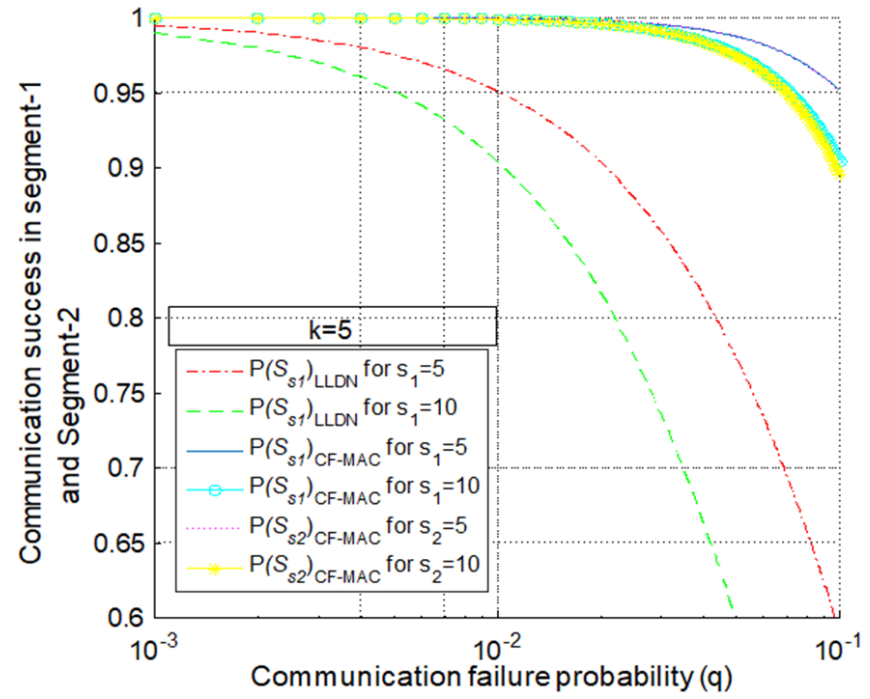

Figure 10: Segment-wise communication success ratio, CF-MAC vs LLDN

is presented in detail in Section 3.2.2, and a ratio is established between the number of time-slots $(h)$ in segment-3 (Figure 3) and total supervisory/alerting traffic nodes $(u)$ affiliated to a cluster. In Figure 9, a ratio between $h$ and $u$ is represented where the time-slots represented on x-axis can accommodate supervisory control nodes represented by bar graphs, while ensuring that $99.9 \%$ of the times the channel access requests from supervisory nodes will not exceed the designated time-slots in the superframe. This allows asynchronous communication to be incorporated in regular frames without affecting the specified QoS.

To evaluate the performance of regulatory control and supervisory control traffic, reliability and channel access delay are considered. The successful communication in superframe segments (details in Section 3.2 and Figure 3 ) for both IEEE802.15.4e LLDN and CF-MAC is presented in Figure 10. In this figure, with the increase in communication failure probability, the communication in segment-1 and segment-2 significantly suffers in LLDN. However, due to the adaptive rescheduling introduced in CF-MAC, the performance decline is much steadier. In Figure 10, At communication failure probability, $q=10^{-2}(99 \%$ 


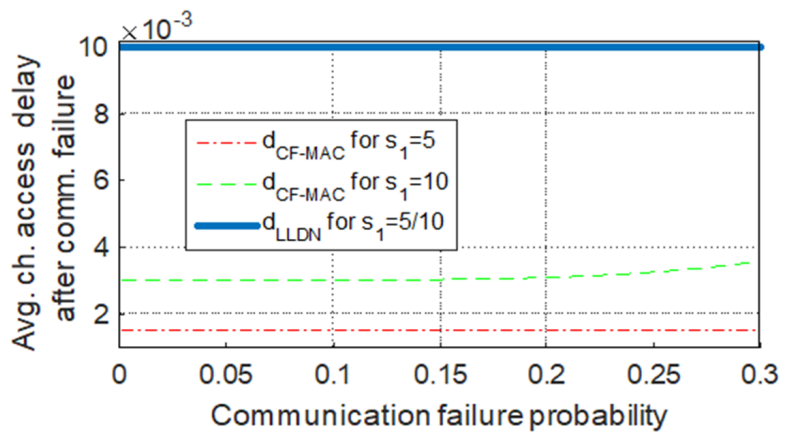

Figure 11: Channel access delay: CF-MAC vs LLDN

PRR ensured from individual sources), the success ratio of entire segment drops to $95 \%$ for segment size $\left(s_{1}\right)$ of 5 and $90 \%$ for $s_{1}$ equal to 10 in case of LLDN. Whereas for the same communication failure probability $\left(q=10^{-2}\right)$, the success ratio for the segment- 1 and segment-2 remains almost 100\% in CF-MAC (for both $\left.s_{1}=5,10 ; s_{2}=5,10\right)$. CF-MAC noticeably improves the communication reliability. However, due to the lower priority of segment-2 nodes, reliability of segment-2 is slightly lower than segment-1. Therefore, it can be seen that the plots for $P\left(S_{s 1}\right)_{C F-M A C}$ for $s_{1}=5$ and $P\left(S_{s 2}\right)_{C F-M A C}$ for $s_{2}=5$ are almost overlapping. Same is observed in plots of $P\left(S_{s 1}\right)_{C F-M A C}$ for $s_{1}=10$ and $P\left(S_{s 2}\right)_{C F-M A C}$ for $s_{2}=10$.

\section{Conclusion and Future Directives}

Three MAC protocols, EE-MAC, CF-MAC and OD-MAC are proposed in this paper. Results have shown that the proposed protocols offer notable improvements in comparison to IEEE 802.15.4e LLDN. For emergency systems, EE-MAC offered a $31 \%$ to $91 \%$ decrease in average delay $\left(d_{\text {success }}\right)$ of emergency communications at the cost of under $7 \%$ increase in delay of non-critical time insensitive communications. CF-MAC offered a notable improvement in reliability along with a $60 \%$ and $85 \%$ improvement in channel access delay in regulatory control and open-loop control traffic. Further to this, the paper also presented process modelling for incorporating asynchronous communications in 
the superframe and proposed a dynamic deadline based scheduling algorithm.

As a future aspect of the presented research, the proposed MAC protocols can be extended for multi-channel scenario with parallel communication streams. Cognitive sensing can also be included to offer improved bandwidth efficiency. Another aspect could be to increase in reliability by incorporating reliability delay trade-off (setting the delay to maximum allowable limit to increase reliability). Suitable energy preservation, sleep scheduling and energy harvesting mechanisms can also be incorporated for overall network lifetime enhancement. To offer better scalability and to diversify the intended application area, adaptive transition mechanism can also be introduced where any of the proposed protocols can be adaptively selected on runtime.

\section{References}

[1] M. Magno, D. Boyle, D. Brunelli, B. O'Flynn, E. Popovici, and L. Benini, "Extended Wireless Monitoring Through Intelligent Hybrid Energy Supply," Industrial Electronics, IEEE Transactions on, vol. 61, pp. 1871-1881, 2014.

[2] T. M. Chiwewe and G. P. Hancke, "A Distributed Topology Control Technique for Low Interference and Energy Efficiency in Wireless Sensor Networks, " Industrial Informatics, IEEE Transactions on, vol. 8, pp. 11-19, 2012.

[3] B. C. Villaverde, S. Rea, and D. Pesch, "InRout A QoS aware route selection algorithm for industrial wireless sensor networks," Ad Hoc Networks, vol. 10, pp. 458-478, 2012.

[4] F. Dobslaw, T. Zhang and M. Gidlund, "QoS-Aware Cross-Layer Configuration for Industrial Wireless Sensor Networks," in IEEE Transactions on Industrial Informatics, vol. 12, no. 5, pp. 1679-1691, Oct. 2016.

[5] M. Raza, N. Aslam, H. Le-Minh, S. Hussain, Y. Cao and N. M. Khan, "A Critical Analysis of Research Potential, Challenges and Future Directives 
in Industrial Wireless Sensor Networks," in IEEE Communications Surveys \& Tutorials, vol. PP, no. 99, pp. 1-1. doi: 10.1109/COMST.2017.2759725

[6] P. Zand, S. Chatterjea, K. Das, and P. Havinga, "Wireless industrial monitoring and control networks: The journey so far and the road ahead," Journal of sensor and actuator networks, vol. 1, pp. 123-152, 2012.

[7] L. F. M. de-Moraes and R. S. Silva, "Analysis of multichannel wireless networks With priority-based polling MAC protocols," in Wireless Days (WD), 2014 IFIP, 2014, pp. 1-6.

[8] S. Wei, Z. Tingting, F. Barac, and M. Gidlund, "PriorityMAC: A PriorityEnhanced MAC Protocol for Critical Traffic in Industrial Wireless Sensor and Actuator Networks," Industrial Informatics, IEEE Transactions on, vol. 10, pp. 824-835, 2014.

[9] Utility Industry (Zigbee Smart Energy) — The ZigBee Alliance. Available: http://www.zigbee.org/what-is-zigbee/utility-industry/ (Accessed: January 2018)

[10] S. Petersen and S. Carlsen, "WirelessHART Versus ISA100.11a: The Format War Hits the Factory Floor," Industrial Electronics Magazine, IEEE, vol. 5, pp. 23-34, 2011.

[11] 6loWPAN Active Drafts. Available: https://datatracker.ietf.org/doc/search/ ?name $=6$ loWPAN\&activeDrafts $=$ on\&rfcs $=$ on $($ Accessed: July 2017)

[12] "IEEE Standard for Information technology- Local and metropolitan area networks- Specific requirements- Part 15.4: Wireless Medium Access Control (MAC) and Physical Layer (PHY) Specifications for Low Rate Wireless Personal Area Networks (WPANs)," IEEE Std 802.15.4-2006 (Revision of IEEE Std 802.15.4-2003), pp. 1-320, 2006.

[13] "IEEE Standard for Local and metropolitan area networks-Part 15.4: LowRate Wireless Personal Area Networks (LR-WPANs) Amendment 1: MAC 
sublayer," IEEE Std 802.15.4e-2012 (Amendment to IEEE Std 802.15.4-

[14] M. Raza, H. Le-Minh, N. Aslam, S. Hussain and W. Ellahi, "A control channel based MAC protocol for time critical and emergency communications in Industrial Wireless Sensor Networks," 2017 International Conference on Communication, Computing and Digital Systems (C-CODE), Islamabad,

[16] T. Zheng, M. Gidlund, and J. Akerberg, "WirArb: A New MAC Protocol for Time Critical Industrial Wireless Sensor Network Applications," Sensors Journal, IEEE, vol. PP, pp. 1-1, 2015.

Mohsin Raza is a research assistant at Middlesex University, London and $\mathrm{PhD}$ scholar at Northumbria University, Newcastle, UK. He also worked as a Lecturer

Hoa Le-Minh is a senior lecturer at Northumbria University, UK. Hoa also worked as a research assistant in Siemens, Germany (20022004) and postdoctoral research fellow (2007-2010) in University of Oxford, UK.

Nauman Aslam is a Reader in the Department of Computer Science and 410 Northumbria University, he worked as an Assistant Professor at Dalhousie University, Canada.

Sajjad Hussain is a Lecturer at School of Engineering, University of Glasgow, UK. Prior to this, he was Associate Professor at Capital University of Science 415 